\title{
HYPERSPECTRAL SUPER-RESOLUTION OF LOCALLY LOW RANK IMAGES FROM COMPLEMENTARY MULTISOURCE DATA
}

\author{
M.A. Veganzones ${ }^{1 *}$, M. Simões ${ }^{1,2}$, G. Licciardi $^{1}$, J. Bioucas ${ }^{2}$ and J. Chanussot ${ }^{1,3}$ \\ ${ }^{1}$ GIPSA-lab, Grenoble-INP, Saint Martin d'Hères, France \\ ${ }^{2}$ Instituto de Telecomunicações, Instituto Superior Técnico, Lisbon, Portugal \\ ${ }^{3}$ Faculty of Electrical and Computer Engineering, University of Iceland, Reykjavik, Iceland
}

March 19, 2014

\begin{abstract}
Remote sensing hyperspectral images (HSI) are quite often locally low rank, in the sense that the spectral vectors acquired from a given spatial neighborhood belong to a low dimensional subspace/manifold. This has been recently exploited for the fusion of low spatial resolution HSI with high spatial resolution multispectral images (MSI) in order to obtain super-resolution HSI. Most approaches adopt an unmixing or a matrix factorization perspective. The derived methods have led to state-ofthe-art results when the spectral information lies in a low dimensional subspace/manifold. However, if the subspace/manifold dimensionality spanned by the complete data set is large, the performance of these methods decrease mainly because the underlying sparse regression is severely ill-posed. In this paper, we propose a local approach to cope with this difficulty. Fundamentally, we exploit the fact that real world HSI are locally low rank, to partition the image into patches and solve the data fusion problem independently for each patch. This way, in each patch the subspace/manifold dimensionality is low enough to obtain useful superresolution. We explore two alternatives to define the local regions, using sliding windows and binary partition trees. The effectiveness of the proposed approach is illustrated with synthetic and semi-real data.
\end{abstract}

\section{Introduction}

In recent years, there has been a huge improvement of spectral and spatial resolutions in the design of remote sensing sensors. However, it seems that it is not possible to achieve both of them simultaneously. This is due, on the

*This work was supported by the Delegation Générale de l'Armement (DGA) under contract PDE2012600079. 
one hand, to the system trade-off related to data volume and signal-to-noise ratio (SNR) limitations and, on the other hand, to the specific requirements of different applications [1]. Then, there is a need for super-resolution techniques that fuse high spectral resolution images, such as hyperspectral images (HSI), with high spatial images such as multispectral images (MSI) or panchromatic images, in order to obtain high spectral and spatial (super-resolution) images.

Recently, some techniques dedicated to the fusion of HSIs and MSIs have been proposed. A general trend is to associate this problem with either linear spectral unmixing, which assumes that the underlying data can be described by a mixture of a relatively small number of "pure" spectral signatures, corresponding to the materials present in the scene or, with the learning of a spectral dictionary that codifies the information present on the images. Since both HSIs and MSIs capture the same scene, the underlying materials (the so-called endmembers) or the dictionaries should be the same. Therefore, the spectral information extracted from one of the images should also be able to explain the other one. Due to the high spectral resolution of the HSIs, the endmembers or the dictionary are extracted from these data, and are then used to reconstruct the MSI. Since MSIs have high spatial resolution, the final reconstructed image will be at super-resolution.

\section{$1.1 \quad$ Related work}

Zurita et al. [2], introduced one of the first unmixing-based approaches to the fusion of remote sensing multiband images. A related approach is proposed in [3], where a very high-resolution hyperspectral image is estimated from a lowerresolution hyperspectral image and a high-resolution RGB image. The method starts by identifying an unmixing matrix used to represent the hyperspectral spectra and then uses this matrix in conjunction with the RGB input to compute, via sparse regression, representation coefficients for the high-resolution hyperspectral image. This methodology can be viewed as a factorization of the input into a mixing matrix and a set of maximally sparse coefficients. An approach with similar flavour is proposed in [4]. The main difference is that the mixing matrix is replaced by a dictionary learnt using a non-negative matrix factorization with sparsity regularization on the code. In [5], the hyperspectral data is unmixed via the K-SVD algorithm, and the multispsectral data is reconstructed using orthogonal matching pursuit to induce sparsity. Authors in [1] proposed a method where two dictionaries were learned from the two different data, and then used a dictionary-pair learning method to establish the correspondence between them. A similar and older technique introduced in [6] alternately unmixes both sources of data to find the signatures and the abundances of the endmembers.

\subsection{Contribution}

In real world HSI, it is very likely that in a small spatial neighbourhood the number of different materials is small, i.e., these images are locally low rank. We 
take advantage of this property to propose a local super-resolution methodology. Firstly, we propose two approaches, one using a square sliding window of fixed size and a second using a binary partition tree representation, to obtain a set of patches from the image, such that the set of pixels with indices in each patch span a subspace of lower dimensionality than that of the whole image. Secondly, we exploit the locally low rank property of the pixels of each patch in two different ways: by using an endmembers induction algorithm (EIA) to find the low rank subspace spanned by the spectral signatures of the materials present in each patch; and, by a singular value decomposition (SVD)-based approach to find the local low-rank subspace of the data of each patch. The proposed methodology does not need to estimate the spatial blur, as far as it is constant across bands.

\subsection{Outline}

The remainder of the paper is organized as follows: in Sec. 2 the super-resolution problem is formulated, in Sec. 3 the proposed local super-resolution methodology is introduced and finally, in Sec. 4 experimental results and some conclusion remarks are provided.

\section{Problem formulation}

Let $\mathbf{X} \in \mathbb{R}^{n_{h} \times n}$ denote a HSI with $n_{h}$ spectral bands (rows of $\mathbf{X}$ ) and $n=n_{x} \times n_{y}$ pixels (columns of $\mathbf{X}$ ). We may interpret $\mathbf{X}$ either as a collection of $n_{h}$ images (or bands) of size $n_{x} \times n_{y}$, each one associated to a given wavelength interval, or as a collection of $n$ spectral vectors of size $n_{h}$, each one associated with a given pixel. In this work, we are concerned with the estimation of $\mathbf{X}$, which we term the original HSI, from two degraded observations of $\mathbf{X}$ : a) a low spatial resolution HSI, $\mathbf{Y}_{h} \in \mathbb{R}^{n_{h} \times\left(n_{x} / d\right) \times\left(n_{y} / d\right)}$, where $d>1$ denotes a spatial downsampling factor, and b) a MSI, $\mathbf{Y}_{m} \in \mathbb{R}^{n_{m} \times n_{x} \times n_{y}}$, where $n_{m} \ll n_{h}$. We assume that $\mathbf{Y}_{h}$ is generated as

$$
\mathbf{Y}_{h}=\mathbf{X B M}+\mathbf{N}_{h}
$$

where $\mathbf{B} \in \mathbb{R}^{n \times n}$ is a matrix modeling band independent sensor blur, $\mathbf{M} \in$ $\mathbb{R}^{n \times\left(n / d^{2}\right)}$ is a masking matrix accounting for spatial downsampling of size $d$ on both spatial dimensions, and $\mathbf{N}_{h}$ is an additive perturbation. Concerning the MSI, $\mathbf{Y}_{m}$, we assume the generation model

$$
\mathbf{Y}_{m}=\mathbf{R X}+\mathbf{N}_{m}
$$

where the matrix $\mathbf{R} \in \mathbb{R}^{n_{m} \times n_{h}}$ holds in its columns the $n_{h}$ spectral responses of the multispectral sensor, and $\mathbf{N}_{m}$ is an additive perturbation.

Let us suppose that it is possible to learn a dictionary $\mathbf{D} \in \mathbb{R}^{n_{h} \times n_{d}}$ from the hyperspectral image $\mathbf{Y}_{h}$, and that the columns $\mathbf{X}$, denoted by $\mathbf{x}_{i}$, for $i=$ $1, \ldots, n$, may be sparsely represented as linear combinations of the columns of 
D. That is, given $\mathbf{x}_{i}$ for $i \in \mathcal{S}$, there is a sparse vector $\boldsymbol{\alpha}_{i} \in \mathbb{R}^{n_{d}}$ (i.e., only a few components of $\boldsymbol{\alpha}_{i}$ are non-zero) such that:

$$
\mathbf{x}_{i}=\mathbf{D} \boldsymbol{\alpha}_{i} .
$$

By replacing (3) in (2), we obtain:

$$
\mathbf{Y}_{m}=\mathbf{R D A}+\mathbf{N}_{m},
$$

where $\mathbf{A} \equiv\left[\boldsymbol{\alpha}_{1}, \ldots, \boldsymbol{\alpha}_{n}\right]$, is often termed as code in dictionary learning and sparse regression applications. If equation (4) can be solved with respect to $\mathbf{A}$, then we may plug its solution into (3) and thereby obtain an estimate of $\mathbf{X}$.

The success of a dictionary-based approach depends fundamentally on the ability to solve (4) with respect to $\mathbf{A}$. The difficulty in solving this system comes from the fact that the system matrix $\mathbf{R D} \in \mathbb{R}^{n_{m} \times n_{d}}$ is often fat, i.e., $n_{m}<n_{d}$, yielding an undetermined system of equations. A typical multispectral sensor has less than 10 bands, quite often 4 in the wavelength interval $[0.4,2.5]$ microns where most hyperspectral sensors operate, whilst $n_{d}$ is often of the order of a few tens. The ill-posedness of (2) when $n_{m}<n_{d}$ may be cured by exploiting the sparsity of the codes $\boldsymbol{\alpha}_{i}$, for $i \in \mathcal{S}$, which opens the door to all sort of sparse regression techniques, many of them recently introduced in compressive sensing [7] applications. Nevertheless, we still face a difficulty: in hyperspectral applications, the columns of $\mathbf{D}$ tend to be highly correlated, implying that the mutual coherence of the columns of RD is close to 1 . This makes (2) ill-posed even when codes $\boldsymbol{\alpha}_{i}$, for $i \in \mathcal{S}$, are sparse [8, 9].

In the next section, we introduce two local dictionary-based techniques conceived to cope with the ill-posedness with origin in the matrix system RD. The main idea, in the vein of the local approaches to image restoration, is to decompose the HSI into patches and build patch-dependent dictionaries such that $n_{m} \geq n_{d}$ in each patch. Thereby, the inverse problem of solving (2) in each patch is well-posed.

\section{Local dictionary based HSI super-resolution}

\subsection{Local spatial patches definition}

Let $\mathcal{P}_{j}$ denote the set of patches obtained from an image, $\mathbf{Y}_{h}$, such that the set of pixels with indices in each patch, $\mathbf{Y}_{j} \equiv\left[\mathbf{y}_{i}, i \in \mathcal{P}_{j}\right]$, span a subspace of lower dimensionality than that of $\mathbf{Y}_{h}$. Next, we introduce two approaches to obtain such patches. The first approach uses a sliding window and the second approach relies on a binary partition tree (BPT) representation [10].

\subsubsection{Using a sliding window of fixed size}

The sliding window methodology has been broadly used in image processing. It consists in defining a square window of fixed size so the pixels lying inside the window form a patch. The window then slides over the whole image in a 
standard zig-zag way, be it with some overlapping or not, eventually covering the whole image and defining the image patches, $\mathcal{P}_{j}$.

\subsubsection{Using a BPT representation}

The BPT is a hierarchical region-based representation of an image in a tree structure [10]. In the BPT representation, the leaf nodes correspond to an initial partition of the image, which can be the individual pixels, or a coarser segmentation map. From this initial partition, an iterative bottom-up region merging algorithm is applied until only one region remains. This last region represents the whole image and corresponds to the root node. All the nodes between the leaves and the root result of the merging of two adjacent children regions.

Two notions are of prime importance when defining a BPT, the region model, $\mathcal{M}_{\mathcal{R}}$, which specifies how a region $\mathcal{R}$ is modelled, and the merging criterion, $\mathcal{O}\left(\mathcal{M}_{\mathcal{R}_{\alpha}}, \mathcal{M}_{\mathcal{R}_{\beta}}\right)$, which is a distance measure between the region models of any two regions $\mathcal{R}_{\alpha}$ and $\mathcal{R}_{\beta}$. Each merging iteration involves the search of the two neighbouring regions which achieve the lowest pair-wise similarity among all the pairs of neighbouring regions in the current segmentation map. Those two regions are consequently merged. To build the BPT representation from the hyperspectral image $[11,12,13], \mathbf{Y}_{h}$, we use the first-order parametric model $\mathcal{M}_{\mathcal{R}}:$

$$
\mathcal{M}_{\mathcal{R}} \stackrel{d}{=} \overline{\mathbf{x}}=\frac{1}{N_{\mathcal{R}}} \sum_{i=1}^{N_{\mathcal{R}}} \mathbf{x}_{i},
$$

where $N_{\mathcal{R}}$ is the number of pixels on the region; and, in order to merge regions, the spectral angle distance:

$$
\mathcal{O}\left(\mathcal{M}_{\mathcal{R}_{\alpha}}, \mathcal{M}_{\mathcal{R}_{\beta}}\right) \stackrel{d}{=} d_{\mathrm{SAM}}\left(\overline{\mathbf{x}}_{\alpha}, \overline{\mathbf{x}}_{\beta}\right)=\arccos \left(\frac{\overline{\mathbf{x}}_{\alpha} \overline{\mathbf{x}}_{\beta}}{\left\|\overline{\mathbf{x}}_{\alpha}\right\|\left\|\overline{\mathbf{x}}_{\beta}\right\|}\right) .
$$

Once the BPT representation is built, the BPT is pruned to achieve a partition of the image such that the regions of the partition define the image patches, $\mathcal{P}_{j}$. We use the vote-based pruning approach from [14], where each region of the $\mathrm{BPT}$ representation is voted by its children. A region receives a number of votes equal to the leaves of a children node if the distance between the region model and the children model is above some threshold. Once all the regions have collected their number of votes, the BPT is pruned so the partition cardinality is maximized constrained to the regions of the partition having received at least half the total number of votes.

\subsection{Local dictionary-based super-resolution}

\subsubsection{EIA-based approach}

Given a set of patches $\mathcal{P}_{j}, j=1, \ldots, P$; for each $\mathcal{P}_{j}$, we identify a mixing matrix $\mathbf{D}_{j}$ by applying an EIA to extract a set of endmembers from the set of pixels 
Table 1: Mean and standard deviation values of the average RMSE, average SAM, ERGAS and average Q quality measures for super-resolution methodologies comparison using the synthetic dataset.

\begin{tabular}{|c|c|c|c|c|c|}
\cline { 3 - 6 } \multicolumn{2}{c|}{} & \multicolumn{4}{c|}{ Synthetic } \\
\cline { 3 - 6 } \multicolumn{2}{c|}{} & RMSE & SAM & ERGAS & Q \\
\hline \multicolumn{2}{|c|}{ HSR-GDL-EIA } & $0.0552 \pm 0.0230$ & $4.7777 \pm 3.5114$ & $4.7583 \pm 1.6699$ & $0.9629 \pm 0.0252$ \\
\hline \multirow{2}{*}{ HSR-LDL-EIA } & Windows & $0.0294 \pm 0.0007$ & $2.3944 \pm 0.0610$ & $2.1254 \pm 0.0450$ & $0.9877 \pm 0.0007$ \\
\cline { 2 - 6 } & BPT & $\mathbf{0 . 0 2 9 0} \pm \mathbf{0 . 0 0 0 8}$ & $\mathbf{2 . 2 0 6 7} \pm \mathbf{0 . 0 7 1 4}$ & $\mathbf{2 . 0 5 7 4} \pm \mathbf{0 . 0 4 4 7}$ & $\mathbf{0 . 9 8 7 8} \pm \mathbf{0 . 0 0 0 8}$ \\
\hline \multirow{2}{*}{ HSR-LDL-SVD } & Windows & $0.0295 \pm 0.0009$ & $2.3877 \pm 0.0751$ & $2.1093 \pm 0.0468$ & $0.9875 \pm 0.0009$ \\
\cline { 2 - 6 } & BPT & $\mathbf{0 . 0 2 8 8} \pm \mathbf{0 . 0 0 0 8}$ & $\mathbf{2 . 1 8 1 0} \pm \mathbf{0 . 0 6 9 1}$ & $\mathbf{2 . 0 4 2 9} \pm \mathbf{0 . 0 5 3 5}$ & $\mathbf{0 . 9 8 8 1} \pm \mathbf{0 . 0 0 0 8}$ \\
\hline
\end{tabular}

$\mathbf{X}_{j}$, identified by the corresponding patch. Having identified the dictionaries, $\mathbf{D}_{j}$, the code $\mathbf{A}$ is estimated by solving the following constrained least squares (CLS) problems:

$$
\min _{\mathbf{A}_{j} \geq 0}\left\|\mathbf{Y}_{m, j}-\mathbf{R D}_{j} \mathbf{A}_{j}\right\|_{F}^{2} \quad j=1, \ldots, P
$$

where $\mathbf{A}_{j} \in \mathbb{R}^{n_{d} \times\left|\mathcal{P}_{j}\right|}$ and $\mathbf{Y}_{m, j} \in \mathbb{R}^{n_{m} \times\left|\mathcal{P}_{j}\right|}$ gathers, respectively, the columns of $\mathbf{A}$ and $\mathbf{Y}_{m}$ with indices corresponding to the pixels $\mathbf{X}_{j}$ in $\mathcal{P}_{j}$. The inequality $\mathbf{A}_{j} \geq 0$ is to be understood in the component-wise sense, and $\|\cdot\|_{F}$ denotes the Frobenius norm. The constraint $\mathbf{A}_{j} \geq 0$ in (5) is used because, in the linear mixing model, the codes $\boldsymbol{A}_{j}$ represent abundances of materials which are necessarily non-negative [15]. It is possible to add the abundances sumto-one constraint, $\mathbf{A}_{j}^{T} \mathbf{I}=\mathbf{1}_{\left|\mathcal{P}_{j}\right|}$, but this constraint is usually dropped due to possible scale model mismatches [9]. We remark that, since our observations are spectral vectors and thus non-negative (apart from the noise contribution), the non-negativity constraint in (5) is equivalent to a form of constrained $\ell_{1}$ regularization and therefore, to some kind of sparsity enforcement (see [16] for the details).

In order to solve (5), we use the SUnSAL algorithm [17], which is an instance of the C-SALSA methodology introduced in [18] to effectively solve a large number of CLS problems sharing the same matrix system. Fig. 1 shows the pseudocode of the proposed HSI Super Resolution via Local Dictionary Learning using EIAs (HSR-LDL-EIA). The algorithm operates on each patch independently. Each patch indexes the set of pixels in the hyperspectral image, $\mathbf{Y}_{h, j}$, and in the multispectral image, $\mathbf{Y}_{m, j}$. From the hyperspectral set of pixels, the subspace matrix, $\mathbf{D}_{j}$, is obtained using any EIA. Then, the SUnSAL algorithm is used to estimate the fractional abundances, $\widehat{\mathbf{A}}_{j}$, from the multispectral set of pixels. The fractional abundances are linearly combined with the estimated subspace to estimate the super-resolution pixels of the patch, $\widehat{\mathbf{X}}_{j}$. The estimated super-resolution pixels of all the patches are combined to form the estimated super-resolution image, $\mathbf{X}$. 


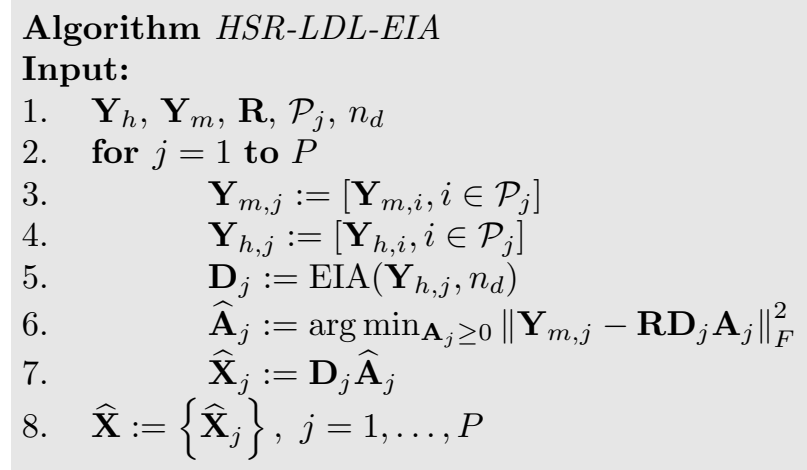

Figure 1: HSI Super Resolution via Local Dictionary Learning using Endmember Induction Algorithms (HSR-LDL-EIA).

Table 2: Mean and standard deviation values of the average RMSE, average SAM, ERGAS and average Q quality measures for super-resolution methodologies comparison using the real Pavia University dataset.

\begin{tabular}{|c|c|c|c|c|c|}
\hline & \multicolumn{4}{|c|}{ Pavia University } \\
\hline & & RMSE & SAM & ERGAS & $\mathrm{Q}$ \\
\hline \multicolumn{2}{|c|}{ HSR-GDL-EIA } & $0.0136 \pm 0.0017$ & $2.9654 \pm 0.3068$ & $2.2056 \pm 0.2994$ & $0.9897 \pm 0.0024$ \\
\hline \multirow{2}{*}{ HSR-LDL-EIA } & Windows & $0.0169 \pm 0.0001$ & $2.9804 \pm 0.0077$ & $2.6302 \pm 0.0082$ & $0.9832 \pm 0.0001$ \\
\hline & BPT & $0.0138 \pm 0.0030$ & $2.6766 \pm 0.4147$ & $2.1924 \pm 0.3118$ & $0.9887 \pm 0.0051$ \\
\hline \multirow{2}{*}{ HSR-LDL-SVD } & Windows & $0.0901 \pm 0.1101$ & $2.7874 \pm 0.1449$ & $15.042 \pm 19.988$ & $0.8445 \pm 0.1345$ \\
\hline & BPT & $0.0153 \pm 0.0015$ & $3.1503 \pm 0.1907$ & $2.9803 \pm 0.3274$ & $0.9844 \pm 0.0037$ \\
\hline
\end{tabular}

\subsubsection{SVD-based approach}

In this method, we carry out the dictionary learning by solving the following optimization problem:

$$
\min \operatorname{rank}\left(\mathbf{D}_{j}\right) \mid \min _{\boldsymbol{\beta}_{j}, \mathbf{D}_{j}}\left\|\mathbf{Y}_{h, j}-\mathbf{D}_{j} \boldsymbol{\beta}_{j}\right\|_{F}^{2} \leq \delta,
$$

where $\mathbf{Y}_{h, j}$ are the observed hyperspectral vectors with indexes in $\mathcal{P}_{j}$, and $\boldsymbol{\beta}_{j}$ are weighting coefficients (the code). The optimization problem (6) aims at finding the dictionary $\mathbf{D}_{j}$ of lower rank, desirably lesser than $n_{m}$, yielding a representation error for $\mathbf{Y}_{h, j}$ smaller than $\delta$. Using the local low rank argument, the range of the subspace spanned by the super-resolution pixels $\mathbf{X}_{j}$ in patch $\mathcal{P}_{j}$ should be close to that of $\mathbf{D}_{j}$ and thus, we may obtain the code for $\mathbf{X}_{j}$ by solving:

$$
\min _{\mathbf{A}_{j}}\left\|\mathbf{Y}_{m, j}-\mathbf{R D}_{j} \mathbf{A}_{j}\right\|_{F}^{2}+\tau\left\|\mathbf{A}_{j}\right\|_{F}^{2} \quad j=1, \ldots, P .
$$

Comparing (7) with (5), we have discarded the non-negativity constraint and have introduced the quadratic term $\tau\left\|\mathbf{A}_{j}\right\|_{F}^{2}$, where $\tau \geq 0$ is a regularization parameter. We have discarded the non-negativity constraint because the columns 


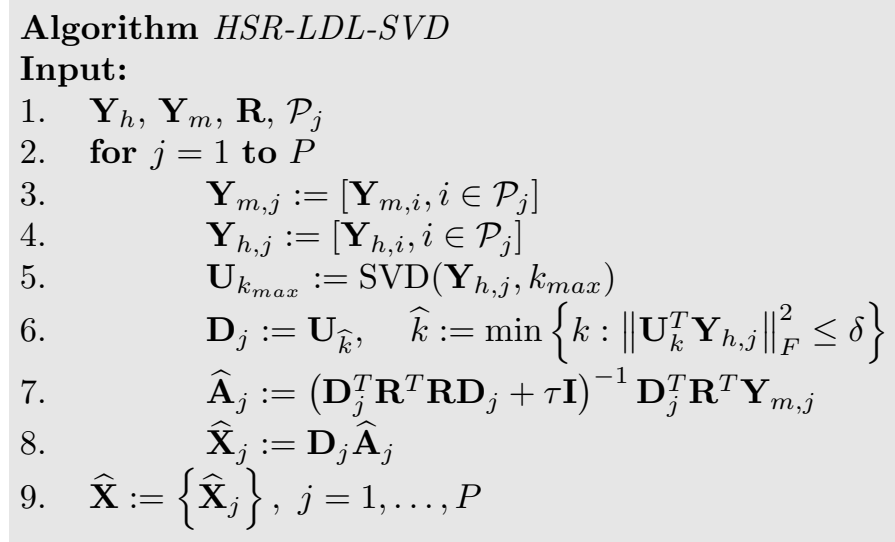

Figure 2: HSI Super Resolution via Local Dictionary Learning using the Singular Value Decomposition (HSR-LDL-SVD).

of $\mathbf{D}_{j}$ are no more interpretable as spectra of pure materials and thus $\mathbf{A}_{j}$ are no more interpretable as abundances. The introduction of a quadratic regularizer aims at improving the noise-amplification we may have owing to the very low singular vectors of $\mathbf{R D}_{j}$.

Although problem (6) is nonconvex, it can easily be solved based on the SVD of $\mathbf{Y}_{h, j}$. Let $\mathbf{U}_{k} \in \mathbb{R}^{n_{h} \times k}$ be a matrix holding the $k$ left singular vectors of $\mathbf{Y}_{h, j}$ ordered by decreasing value of the corresponding singular values. Then, for $\operatorname{rank}\left(\mathbf{D}_{i}\right)=k$, it holds that the solution to:

$$
\min _{\boldsymbol{\beta}_{j}, \mathbf{D}_{j}}\left\|\mathbf{Y}_{h, j}-\mathbf{D}_{j} \boldsymbol{\beta}_{j}\right\|_{F}^{2}=\left\|\mathbf{U}_{k}^{T} \mathbf{Y}_{h, j}\right\|_{F}^{2}
$$

is achieved for $\mathbf{D}_{j}=\mathbf{U}_{k}$ [19]. Therefore, the solution of (6) is given by:

$$
\mathbf{D}_{j}:=\mathbf{U}_{\widehat{k}} \mid \widehat{k}=\arg _{k} \min \left\|\mathbf{U}_{k}^{T} \mathbf{Y}_{h, j}\right\|_{F}^{2} \leq \delta
$$

Fig. 2 shows the pseudocode of the proposed HSI Super Resolution via Local Dictionary Learning using Single Value Decomposition (HSR-LDL-SVD). The algorithm works in a similar way to the HSR-LDL-EIA algorithm. The main difference is that the subspace matrix, $\mathbf{D}_{j}$, is obtained from the $\widehat{k}$ columns of the singular value decomposition of the hyperspectral pixels. Then, the fractional abundances, $\widehat{\mathbf{A}}_{j}$, are calculated from the multispectral pixels using the analytic solution to the regularized inversion problem (8). Finally, the super-resolution pixels of the patch, $\widehat{\mathbf{X}}_{j}$, are calculated; and, the estimated super-resolution pixels of all the patches are combined to form the estimated super-resolution image, $\mathbf{X}$. 


\section{Experimental Results and conclusions}

In order to assess the performance of the proposed local super-resolution methods, we used the following experimental methodology: 1) Given a super-resolution image, $\mathbf{X}$, we simulate a low-spatial resolution hyperspectral image, $\mathbf{Y}_{h}$, by applying a Gaussian blurring $(\mathbf{B})$ and downsampling the blurred image by a factor of four (M). 2) We also simulate a high-spatial resolution multispectral image, $\mathbf{Y}_{m}$, by applying a spectral response matrix $(\mathbf{R})$ to the original super-resolution image. 3) White noise was added to both images, $\mathbf{Y}_{h}$ and $\mathbf{Y}_{m}$, with $30 d b$ and $40 d b$ respectively. 4) Then, we estimate the super-resolution image, $\hat{\mathbf{X}}$, by applying a super-resolution methodology to both images, $\mathbf{Y}_{h}$ and $\mathbf{Y}_{m}$. 5) Finally, we compare the original and the estimated super-resolution images, using the RMSE, the SAM, the ERGAS and the $Q$ quality measures [1].

We compared the two proposed local super-resolution approaches, the HSRLDL-EIA and the HSR-LDL-SVD, to the global approach based on EIAs, HSRGDL-EIA. The SVD-based approach has been defined explicitly for the local approach. Its global version performs poorly and thus, there is no point in showing the comparison. For the proposed local approaches, we also compared the sliding window and BPT-based approaches to obtain the image patches. In the case of the EIA-based approach, we used the Vertex Component Analysis (VCA) EIA [20]. We made the comparison over two datasets:

1. A synthetic image composed of multiple geometric shapes (ellipses and rectangles) of different sizes and orientations, where each geometrical element and the background are formed using a different linear mixture of 5 endmembers from the USGS spectral library, for a total of 45 different endmembers in the image. This image simulates a high spectral variability scenario. In this case, we fixed the number of induced endmembers and selected SVD components to $p=2$ for the local approaches, and $p=45$ for the global one.

2. The well-known Pavia University dataset. This dataset is a real image representing an urban area mainly composed of buildings, urban vegetation and parking lots. The multi-spectral image was simulated using the spectral response of the first three bands of the Ikonos sensor. For the local approaches, we fixed the number of induced endmembers and selected SVD components to $p=3$. For the global approach, this value was set to $p=10$.

Tables 1-2 show the results obtained for the synthetic and Pavia University datasets respectively. For each quality measure, it is shown the mean and standard deviation of Monte Carlo runs. Results show that the local approaches outperform the global one in the synthetic scene, and that the EIA-based local approach does the same in the real scene. From the point of view of the patches definition, the BPT representation shows a slightly higher performance, hinting at the relevance of the patching definition process. However, the windowing approach shows slightly lower standard deviation probably due to the effects 
of noise in the BPT partitioning. The lack of space prevents us from showing the obtained super-resolution images for visual comparison. We encourage the interested reader to access the results extended report at first author's website ${ }^{1}$. We'll further work on the validation of the proposed methodologies and on the study of the patches definition by means of other BPT pruning strategies. We'll also face the problem of estimate the spectral response matrix, $\mathbf{R}$, from the data.

Figs. 3 and 4 show false color representations of the actual Pavia University dataset and the super-resolution estimated images obtained by the compared approaches.

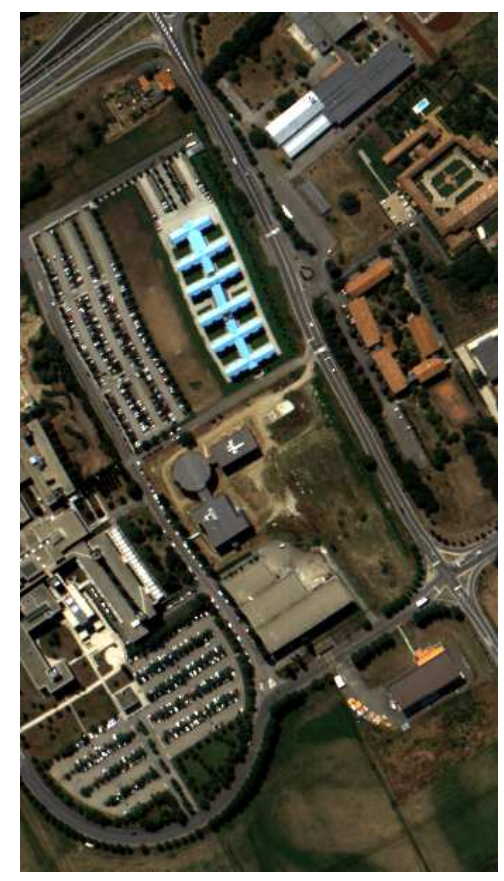

Figure 3: False color representations of the Pavia University dataset.

\section{Acknowledges}

Thanks for funding to Fundação para a Ciência e Tecnologia (FCT), Portuguese Ministry of Science and Higher Education, projects PEst-OE/EEI/0008/2013 and PTDC/EEI-PRO/1470/2012, and grant SFRH/BD/87693/2012.

\footnotetext{
${ }^{1}$ http://www.gipsa-lab.grenoble-inp.fr/page_pro.php?vid=1728
} 


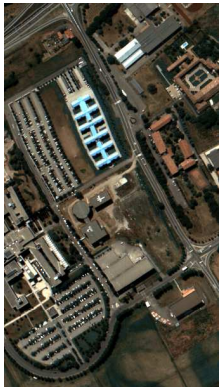

(a)

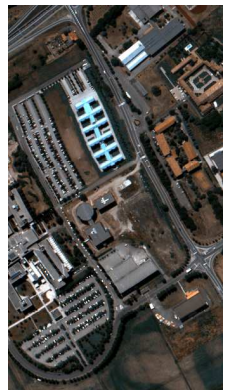

(f)

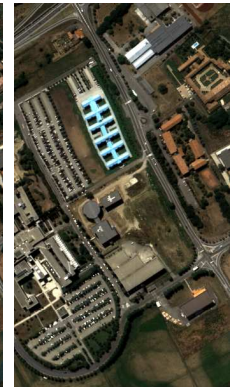

(b)

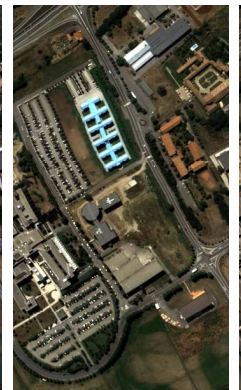

(g)

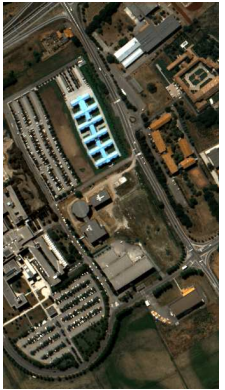

(c)

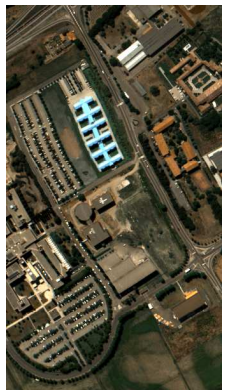

(h)

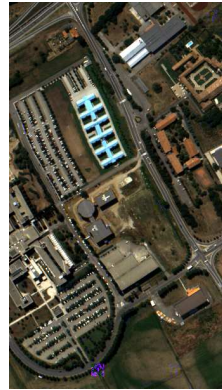

(d)

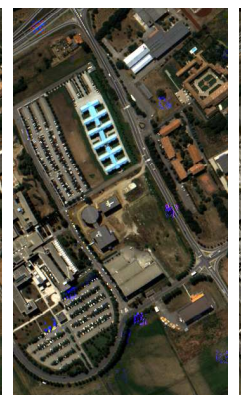

(i)

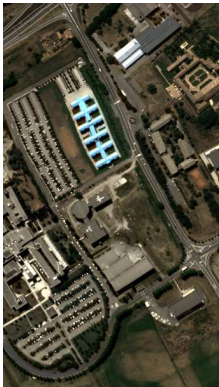

(e)

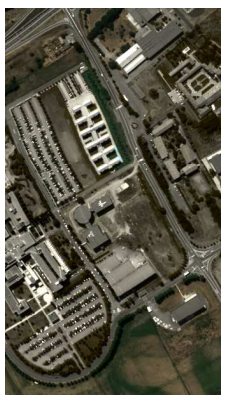

(j)

Figure 4: False color representations of the super-resolution images obtained from the simulated hyperspectral and multispectral observations of the Pavia University dataset. Top and bottom rows respectively show the best and the worst images obtained by: (a,f) HSR-GDL-EIA, (b,g) HSR-LDL-EIA (windows), (c,h) HSR-LDL-EIA (BPT), (d,i) HSR-LDL-SVD (windows) and, (e,j) HSR-LDL-SVD (BPT)

\section{References}

[1] H. Songa, B. Huanga, K. Zhangb, and H. Zhanga, "Spatio-spectral fusion of satellite images based on dictionary-pair learning," Information Fusion, vol. 18, pp. $148-160,2014$.

[2] R. Zurita-Milla, J. Clevers, and M. Schaepman, "Unmixing-based landsat tm and meris fr data fusion," IEEE Geoscience and Remote Sensing Letters, vol. 5, no. 3, pp. 453-457, 2008.

[3] R. Kawakami, J. Wright, Y.-W. Tai, Y. Matsushita, M. Ben-Ezra, and K. Ikeuchi, "High-resolution hyperspectral imaging via matrix factorization," in 2011 IEEE Conference on Computer Vision and Pattern Recognition (CVPR), 2011, pp. 2329-2336. 
[4] A. S. Charles, B. A. Olshausen, and C. J. Rozell, "Learning sparse codes for hyperspectral imagery," IEEE Journal of Selected Topics in Signal Processing, vol. 5, no. 5, pp. 963-978, 2011.

[5] B. Huang, H. Song, H. Cui, J. Peng, and Z. Xu, "Spatial and spectral image fusion using sparse matrix factorization," IEEE Transactions on Geoscience and Remote Sensing, vol. 52, no. 3, pp. 1693-1704, 2014.

[6] N. Yokoya, T. Yairi, and A. Iwasaki, "Coupled nonnegative matrix factorization unmixing for hyperspectral and multispectral data fusion," IEEE Transactions on Geoscience and Remote Sensing, vol. 50, no. 2, pp. 528$537,2012$.

[7] R. G. Baraniuk, "Compressive sensing [lecture notes]," IEEE Signal Processing Magazine, vol. 24, no. 4, pp. 118-121, 2007.

[8] M.-D. Iordache, J. M. Bioucas-Dias, and A. Plaza, "Sparse unmixing of hyperspectral data," IEEE Transactions on Geoscience and Remote Sensing, vol. 49, no. 6, pp. 2014-2039, 2011.

[9] J. M. Bioucas-Dias, A. Plaza, N. Dobigeon, M. Parente, Q. Du, P. Gader, and J. Chanussot, "Hyperspectral unmixing overview: Geometrical, statistical, and sparse regression-based approaches," IEEE Journal of Selected Topics in Applied Earth Observations and Remote Sensing, vol. 5, no. 2, pp. 354-379, 2012.

[10] P. Salembier and L. Garrido, "Binary partition tree as an efficient representation for image processing, segmentation, and information retrieval," IEEE Transactions on Image Processing, vol. 9, no. 4, pp. 561-576, 2000.

[11] S. Valero, P. Salembier, and J. Chanussot, "Comparison of merging orders and pruning strategies for binary partition tree in hyperspectral data," in 17th IEEE International Conference on Image Processing (ICIP), Sept 2010, pp. 2565-2568.

[12] — - "Hyperspectral image representation and processing with binary partition trees," IEEE Transactions on Image Processing, vol. 22, no. 4, pp. 1430-1443, April 2013.

[13] M. Veganzones, G. Tochon, M. Dalla Mura, A. Plaza, and J. Chanussot, "Hyperspectral image segmentation using a new spectral mixture-based binary partition tree-representation," in 20th IEEE International Conference on Image Processing (ICIP), 2013.

[14] G. Tochon, J. Feret, R. Martin, R. Tupayachi, J. Chanussot, and G. Asner, "Binary partition tree as a hyperspectral segmentation tool for tropical rainforests," in 2012 IEEE International Geoscience and Remote Sensing Symposium (IGARSS), 2012, pp. 6368-6371. 
[15] W.-K. Ma, J. Bioucas-Dias, T. Chan, N. Gillis, P. Gader, A. Plaza, A. Ambikapathi, and C. Chi, "A signal processing perspective on hyperspectral unmixing: Insights from remote sensing," IEEE Signal Processing Magazine, vol. 31, no. 1, pp. 67-81, 2014.

[16] A. M. Bruckstein, M. Elad, and M. Zibulevsky, "On the uniqueness of nonnegative sparse solutions to underdetermined systems of equations," IEEE Transactions on Information Theory, vol. 54, no. 11, pp. 4813-4820, 2008.

[17] J. M. Bioucas-Dias and M. A. Figueiredo, "Alternating direction algorithms for constrained sparse regression: Application to hyperspectral unmixing," in IEEE 2nd Workshop on Hyperspectral Image and Signal Processing: Evolution in Remote Sensing (WHISPERS), 2010, pp. 1-4.

[18] M. V. Afonso, J. M. Bioucas-Dias, and M. A. Figueiredo, "An augmented lagrangian approach to the constrained optimization formulation of imaging inverse problems," IEEE Transactions on Image Processing, vol. 20, no. 3, pp. 681-695, 2011.

[19] C. Eckart and G. Young, "The approximation of one matrix by another of lower rank," Psychometrika, vol. 1, no. 3, pp. 211-218, 1936.

[20] J. Nascimento and J. Dias, "Vertex component analysis: a fast algorithm to unmix hyperspectral data," IEEE Transactions on Geoscience and Remote Sensing, vol. 43, no. 4, pp. 898 - 910, april 2005. 